\title{
Measuring stellar magnetic fields from high resolution spectroscopy of near-infrared lines ${ }^{\star}$
}

\author{
F. Leone ${ }^{1}$, W. D. Vacca ${ }^{2,3}$, and M. J. Stift ${ }^{4}$ \\ 1 INAF - Osservatorio Astrofisico di Catania, Via S. Sofia 78, 95123 Catania, Italy \\ 2 Max-Planck-Institut für Extraterrestrische Physik, Postfach 1312, 85741 Garching bei München, Germany \\ 3 Dept. of Astronomy, 601 Campbell Hall, Univ. of California, Berkeley, CA 94520, USA \\ ${ }^{4}$ Institut für Astronomie (IfA), Universität Wien, Türkenschanzstrasse 17, 1180 Wien, Austria
}

Received 22 May 2003 / Accepted 29 July 2003

\begin{abstract}
Zeeman splitting of otherwise degenerate levels provides a straight-forward method of measuring stellar magnetic fields. In the optical, the relative displacements of the Zeeman components are quite small compared to the rotational line broadening, and therefore observations of Zeeman splitting are usually possible only for rather strong magnetic fields in very slowly rotating stars. However, the magnitude of the Zeeman splitting is proportional to the square of the wavelength, whereas rotational line broadening mechanisms are linear in wavelength; therefore, there is a clear advantage in using near-infrared spectral lines to measure surface stellar magnetic fields. We have obtained high resolution $(R \geq 25000)$ spectra in the $15625-15665 \AA$ region for two magnetic chemically peculiar stars, viz. HD 176232 and HD 201601, and for the suspected magnetic chemically peculiar star HD 180583, as part of a pilot study aimed at determining the accuracy with which we can measure stellar magnetic fields using the Zeeman splitting of near-infrared lines. We confirm that in principle the magnetic field strength can be estimated from the magnetic intensification of spectral lines, i.e. the increase in equivalent width of a line over the zerofield value. However, due to line blending as well as the dependence of this intensification on abundance and field geometry, accurate estimates of the magnetic field strengths can be obtained only by modelling the line profiles by means of spectral synthesis techniques. Using this approach, we find a $1.4 \mathrm{kG}$ magnetic field modulus in HD 176132 and an upper limit of $0.2 \mathrm{kG}$ in HD 180583. The very weak infrared lines in the spectrum of HD 201601 are consistent with a $3.9 \mathrm{kG}$ field modulus estimated from the splitting of the Fe II $6149.258 \AA$ line seen in an optical spectrum. Finally, we would like to draw attention to the fact that there are no sufficiently detailed and reliable atomic line lists available for the near-infrared region that can be used in high resolution work; a large fraction of the features observed in our spectra remains to be identified.
\end{abstract}

Key words. line: formation - line: profiles - stars: magnetic fields - stars: chemically peculiar stars: individual: HD 176232- stars: individual: HD 201601

\section{Introduction}

Magnetic fields play a fundamental role in stellar physics across the entire Hertzsprung-Russell (HR) diagram. They have been invoked to explain phenomena such as non-thermal radio emission in O-B stars (e.g., Rauw et al. 2002) and jets in young stellar objects (e.g., Goodson et al. 1999); they are responsible for activity in the Sun and in late-type stars (e.g., Montesinos et al. 2001), and can reach huge strengths in degenerate stars (e.g., Liebert et al. 2003). Despite the importance of stellar magnetic fields, it often proves rather difficult to obtain a direct measure of their strength. The most commonly employed method for that purpose is based on the Zeeman effect, whereby the $(2 J+1)$-fold degeneracy of the fine structure levels of the various spectroscopic terms is lifted by a

Send offprint requests to: F. Leone,

e-mail: fleone@ct.astro.it

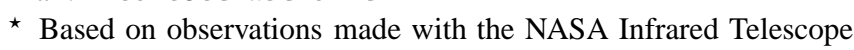
Facility. magnetic field. This results in the splitting of lines into their Zeeman components and the polarisation of these components. Therefore, spectropolarimetric observations of a line can, in principle, yield an estimate of the magnetic field strength and its direction. However, the circular polarisation signal, characteristic of the $\sigma$ Zeeman components of a split line, and the linear polarisation signal, characteristic of the $\pi$ components, both depend strongly on the magnetic field direction, and can cancel to a large degree when fields change sign and orientation on small spatial scales. Sunspots are an example of magnetic structures whose polarimetric signal is barely detectable in the integrated light over the visible hemisphere. As a result, with just a few exceptions, only the large scale magnetic fields of Magnetic Chemically Peculiar (MCP) stars have been measured by means of spectropolarimetry (e.g., Babcock 1958).

Zeeman splitting of a line, on the other hand, provides the most direct method for the detection and measurement of stellar magnetic fields and displays little sensitivity to the mean field orientation. The average wavelength displacement 
of the $\sigma$ components from the central line position $\lambda_{0}$ (in $\AA$ ) is given by

$\Delta \lambda=4.67 \times 10^{-13} g_{\mathrm{eff}} \lambda_{0}^{2} B$

where $g_{\text {eff }}$ is the effective Lande factor and $B$ is the magnetic field strength expressed in Gauss. From this relation it is clear that it is not always possible to measure stellar magnetic field strengths from optical spectra. For example, at $\lambda=5000 \AA$ and with $B=10 \mathrm{kG}$, the displacement of the $\sigma$ component in a simple Zeeman triplet $\left(g_{\text {eff }}=1.0\right)$ is $0.12 \AA$, a value comparable to line broadening due to a rotational velocity of $v_{\mathrm{e}} \sin i \approx$ $7 \mathrm{~km} \mathrm{~s}^{-1}$. Lines with more "favourable" Zeeman patterns (i.e, with larger effective Lande factors and with $\pi$ and $\sigma$ components respectively tightly grouped together) can give rise to separations about a factor of 3 larger than the above estimate. Nevertheless, only about fifty MCP stars whose projected rotational velocities are sufficiently small and whose magnetic fields are quite strong have been observed with high enough resolution to separate their spectral lines into their Zeeman components (see e.g., Mathys et al. 1997).

Because the displacement of the Zeeman components is proportional to the square of the wavelength, while the nonmagnetic line broadening increases only linearly in wavelength, disentangling magnetic effects from other broadening mechanisms becomes much easier with high resolution spectroscopic observations in the near-infrared, rather than the optical, wavelength region. The first attempt to directly measure the solar magnetic field strength from the Zeeman splitting of a near-infrared line was carried out by Harvey \& Hall (1975). They observed the Fe I $3 d^{6} 4 s 5 s^{7} D_{1}-3 d^{6} 4 s 5 p^{7} D_{1}^{o} 15648.510 \AA$ line in order to take advantage of the large effective Landé factor $\left(g_{\text {eff }}=2.97\right)$ for this transition. This line has become a popular target for subsequent investigations of magnetic field strengths, via Zeeman splitting, in studies of the sun and cool stars. A comparison of the sensitivity of diagnostic methods based on observations in the visible, near-infrared, and midinfrared ranges is given by Solanki (1994). A review of the use of near-infrared observations to estimate stellar magnetic field strengths is given by Saar (1994).

Gondoin et al. (1985) attempted to measure stellar magnetic fields from near-infrared spectral lines, applying Robinson's method (1980) which is based on the comparison between magnetically sensitive and insensitive lines. They found that the accuracy of this method is limited by the presence of blends and suggested that a better estimate of the stellar magnetic field could be obtained by comparing the profiles of magnetically sensitive lines with synthetic profiles. In this paper, we investigate the possibility of determining stellar magnetic field strengths via a comparison between high resolution near-infrared spectroscopic observations and synthetic spectra. The latter are calculated with COSSAM (Codice per la Sintesi Spettrale nelle Atmosphere Magnetiche), a Stokes code described in Stift (2000). In the present study we have carried out high-resolution spectroscopy of the 15 625-15665 ^̊ region for two MCP stars, viz. HD 176232 and HD 201601, and for the suspected MCP star HD 180583. In Sect. 2, we briefly review the properties of MCP stars. The near-infrared and optical observations of the three MCP stars chosen for this pilot study are described in Sect. 3. The description of the line modelling and of the effects of a magnetic field on equivalent widths is given in Sect. 4. The results derived from fitting the synthetic spectra to the observed spectra are presented in Sect. 5. Our conclusions are given in Sect. 6.

\section{Magnetic chemically peculiar stars}

MCP stars are found at spectral types between late B and early $\mathrm{F}$ and are characterised by at least three unusual properties: a) certain chemical elements exhibit overabundances with respect to the solar values by factors of up to $10^{6}$ or underabundances by factors of up to 100 ; b) magnetic fields are organised on a large scale with typical strengths between $1-30 \mathrm{kG}$ (integrated over the visible hemisphere) as inferred from the Zeeman effect; and c) the magnetic field strength, luminosity and spectral lines vary with the same period. The longitudinal fields (the component of the integrated magnetic field along the line of sight) often reverse their sign. Typical periods are 2-10 days. As a class, MCP stars are slow rotators, with rotational velocities about three times smaller than those of main sequence stars of the same spectral type. These properties are generally explained by assuming a predominantly dipolar magnetic field, whose symmetry axis is not aligned with the rotational axis of the star (Babcock 1949), and a non-homogeneous distribution of the respective over- and under-abundant elements over the stellar surface. Thus, magnetic, spectroscopic and photometric variations are the direct consequence of stellar rotation. In order to explain abundance anomalies, Michaud (1970) proposed selective levitation of certain ions by the radiation field, which would lead to surface overabundances but possibly also depletion, depending on where the radiative accelerations exceed gravity; other ions would sink, which would result in underabundances.

\section{Observations and data reduction}

High resolution near-infrared spectra of the MCP stars HD 176232, HD 180583 and HD 201601 were obtained with CSHELL on the NASA Infrared Telescope Facility on Mauna Kea. CSHELL is a 1-5.5 micron long-slit, single-order echelle spectrograph employing a $256 \times 256 \mathrm{InSb}$ array; further details of the instrument are given by Greene et al. (1993). HD 201601 was observed on 04 October 1999; HD 176232 and HD 180583 were observed on 02 September 2000. All objects were observed in the so-called "pair-mode", in which the object was positioned at two separate locations ("A" and "B") on the slit and moved from one to the other after each exposure. The airmass for all observations was fairly low $(X<1.3)$ and the position angle of the slit was set to $0^{\circ}$. To obtain the highest resolution afforded by the instrument, the $0.5^{\prime \prime}$ slit was used for all observations. The resolutions achieved, as measured from the widths of the lines seen in exposures of the internal arc lamps (used for wavelength calibration), are estimated to be $R \sim$ 25000 (corresponding to a $F W H M$ of $\sim 0.6 \AA$, or 4.3 pixels) for the October 1999 data and $R \sim 29000$ (FWHM $0.5 \AA$, or 3.5 pixels) for the September 2000 data. The grating angle was set such that the Fe I $15648.510 \AA$ line was located 
Table 1. Observing details.

\begin{tabular}{clcccccc}
\hline \hline $\begin{array}{c}\text { Star } \\
\text { HD }\end{array}$ & $\begin{array}{c}\text { Spect. } \\
\text { Type }^{a}\end{array}$ & \multicolumn{1}{c}{$\mathrm{HJD}^{b}$} & $\begin{array}{c}t_{\exp }^{c} \\
(\mathrm{sec})\end{array}$ & $N^{d}$ & $R^{e}$ & $X^{f}$ & $<S / N>^{g}$ \\
\hline 176232 & A6 Sr & 2451794.812 & 2160 & 18 & 29000 & 1.06 & 380 \\
180583 & F7II & 2451794.894 & 1920 & 16 & 29000 & 1.31 & 490 \\
201601 & A9 SrEu & 2451455.753 & 1920 & 32 & 25000 & 1.02 & 400 \\
201601 & & 2451773.569 & 400 & 1 & 144000 & 1.38 & 230 \\
\hline
\end{tabular}

${ }^{a}$ The spectral type of HD 180583 is from Gray et al. (2001a). Spectral types

and peculiarity classes of HD 176232 and HD 201601 are from Renson et al. (1991).

${ }^{b}$ Heliocentric Julian Date.

${ }^{c}$ Total exposure time in seconds.

${ }^{d}$ Number of individual spectra obtained.

${ }^{e}$ Spectral resolution

${ }^{f}$ Mean airmass for the observations

${ }^{g}$ Mean (statistical) signal-to-noise ratio of the observed spectrum

approximately at the center of the array; the full wavelength range covered was approximately $15625-15665 \AA$. Sixteen A-B pairs were obtained for HD 201601; 9 pairs were obtained for HD 176232 and 8 were obtained for HD 180583. Exposure times for individual integrations were $60 \mathrm{~s}$ each for HD 201601 and $120 \mathrm{~s}$ each for HD 176232 and HD 180583. Flat field and arc frames were obtained immediately after each set of object frames using the flat and arc lamps internal to CSHELL. Details of the observations are listed in Table 1.

The data were reduced with IRAF. The flat field frames were normalised by the mode of the values in the frames, median combined, and then multiplied by a correction function to account for the differences in the slit illumination between the lamps and the sky. For the science frames, each "B" frame was subtracted from the corresponding "A" frame, and the result was divided by the normalised, illumination-corrected flatfield. The spectra were extracted using an aperture of 12 pixels $\left(2.4^{\prime \prime}\right)$ for the October 1999 data and 10 pixels (2.0") for the September 2000 data and then wavelength calibrated. The extracted one-dimensional spectra were then normalised (with the IRAF routine continuum) by fitting a low order cubic spline to the data over regions expected to be representative of the continuum. Tests indicate that, due to the rejection algorithm employed and the iterative nature of the fitting routine, changing the sets of data points used to represent the continuum levels results in systematic variations in the output normalised spectra of typically less than $0.5 \%$. The normalised spectra were then median combined. The uncertainties on each median spectrum as a function of wavelength were estimated from the rms deviations of the $N$ input spectra about the median, $\sigma(\lambda)=\operatorname{rms}(\lambda) / \sqrt{N}$. Our estimate of the uncertainties (and signal-to-noise ratio) in each spectrum includes Poisson noise from the source flux and the errors associated with fitting the cubic spline through the selected continuum points for the normalisation. The signal-to-noise ratios of the flat fields were substantially larger than those of the individual source frames and therefore should contribute very little noise to the reduced spectra. Other possible systematic effects are not included in our uncertainty estimates. The signal-to-noise ratio for the final median spectrum of each star, listed in Table 1, was derived from the $\sigma(\lambda)$ values and and represents an average over the entire wavelength range of the spectrum. The final spectra were then shifted in radial velocity to the heliocentric rest frame.

Telluric corrections were estimated using the atmospheric modelling code ATRAN by Lord (1992). The observed wavelength range contains very few atmospheric absorption features, and those present are extremely weak (less than $1 \%$ of the continuum). We corrected the spectra by dividing by the predicted telluric spectrum generated by ATRAN for typical conditions on Mauna Kea and the airmass at which the observations were obtained. The final wavelength-calibrated and normalised spectra are shown together with the predicted telluric absorption spectra in Fig. 1.

An optical spectrum of HD 201601 was obtained on 17 August 2000 with the high resolution spectrograph $(S A R G)$ (Gratton et al. 2003) at the $3.55 \mathrm{~m}$ Telescopio Nazionale Galileo (TNG) at the Observatorio del Roque de los Muchachos (La Palma, Spain). The spectrum covers the region from $4600 \AA$ to about $7000 \AA$ with a resolution of $R=144000$. The spectrum was reduced using standard reduction procedures for spectroscopic observations within the NOAO/IRAF package and then normalised. The final $S / N$ ratio in the Fe II $6149.258 \AA$ line region was larger than 230 .

\section{Modelling the 15625-15665 A spectral region}

The 15625-15665 $\AA$ spectral region for the selected stars has been modelled by means of COSSAM, the Codice per la Sintesi Spettrale nelle Atmosphere Magnetiche described in Stift (2000) and in Wade et al. (2001). COSSAM is an LTE line synthesis code in polarised light - written in Ada95, fully object oriented and parallelised - that can model blended Stokes spectra over large wavelength intervals, both for the sun and for dipolar field geometries in MCP stars. Anomalous Zeeman splitting is treated in detail and direct opacity sampling is carried out over the $\sigma_{-}$, the $\sigma_{+}$, and the $\pi$ components separately. The radiative transfer equation is solved (including magnetooptical effects) with the Zeeman-Feautrier method of 

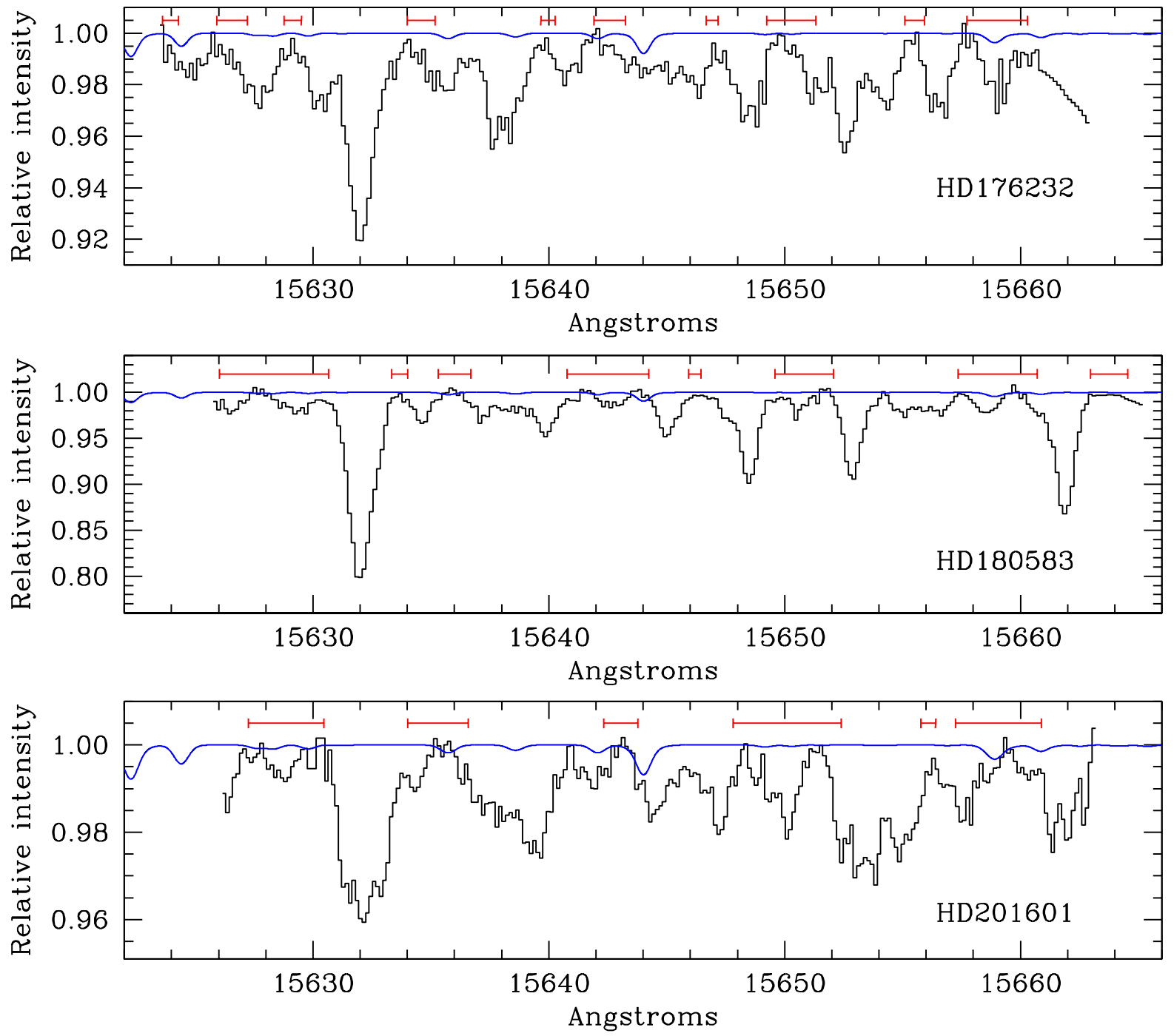

Fig. 1. IRTF spectra of program stars (histograms) shifted in velocity to the respective rest frames. Solid lines represent the telluric atmospheric absorption spectra computed with the ATRAN code (Lord 1992). The data sections used to estimate the stellar continuum (for the normalisation) of HD 176232 and HD 201601 are indicated at the top of the respective panels (see text). Note the different scales for the $y$ axes.

Auer et al. (1977). COSSAM relies on continuous opacities generated by ATLAS9 (Kurucz 1993) since the ATLAS9 models appear to give a very realistic description of the structure of a line-blanketed stellar atmosphere and account for the opacity of about $5.8 \times 10^{7}$ lines. It has been shown that ATLAS9 correctly reproduces the observed UV-to-optical flux distribution and the Balmer line profiles not only for solar composition stars (Castelli \& Kurucz 1994) but also for MCP stars (Leone \& Manfrè 1996). Metal opacity is taken into account by means of Opacity Distribution Functions (ODFs) tabulated for multiples of the solar metallicity. In Table 2 we list the parameters adopted in the computation of the atmospheric models.

It is not possible to model the surface magnetic field structure completely due to the lack of phase coverage. We must content ourselves with measuring the mean magnetic field modulus, averaged over the visible stellar disk, without taking into account the detailed magnetic field configuration. Only with observations available over the whole rotational phase would it be possible to infer the magnetic field geometry (Landstreet \& Mathys 2000).

\subsection{The solar case}

In order to test the quality of the available near-infrared atomic line data used in our models, we have tried to reproduce the solar spectrum obtained by Biémont, and available from the BASS2000 web site ${ }^{1}$. We adopted the atomic line list given by Kurucz (1995) assuming, if possible, the oscillator strengths given in the NIST database. We identified the strongest features present in the spectrum as due to neutral iron. We found however that neither the absolute depths nor the relative strengths of these Fe lines are correctly reproduced with the canonical solar iron abundance of $\log (\mathrm{Fe} / \mathrm{H})=-4.33$. Therefore, we chose to adopt the oscillator strengths derived by Melendez \& Barbuy (1999), who adjusted the oscillator strengths such as to match the observed solar spectrum. Figure 2 shows our synthetic

\footnotetext{
${ }^{1}$ http://bass2000.obspm.fr/home.php
} 




Fig. 2. Solar spectrum in the 15 625-15 $665 \AA$ A range (histogram) and results of our synthetic spectrum calculations (solid line).

Table 2. Adopted and derived stellar parameters.

\begin{tabular}{cccccc|cccc}
\hline \hline $\begin{array}{c}\text { Star } \\
\mathrm{HD}\end{array}$ & $\begin{array}{c}T_{\text {eff }} \\
\mathrm{K}\end{array}$ & $\begin{array}{c}\log g \\
{[\mathrm{cgs}]}\end{array}$ & $\begin{array}{c}\xi \\
\mathrm{km} \mathrm{s}^{-1}\end{array}$ & $\mathrm{[ODF}^{a}$ & $\operatorname{Ref}^{b}$ & $\begin{array}{c}B_{\mathrm{s}}{ }^{c} \\
\mathrm{kG}\end{array}$ & $\begin{array}{c}\Theta^{d} \\
\mathrm{deg}\end{array}$ & $\begin{array}{c}v_{\mathrm{e}} \sin (i) \\
\mathrm{km} \mathrm{s}^{-1}\end{array}$ & $\begin{array}{c}R V^{e} \\
\mathrm{~km} \mathrm{~s}^{-1}\end{array}$ \\
\hline 176232 & 7550 & 4.2 & 0.0 & 0.0 & 1 & $1.4 \pm 0.1$ & $55 \pm 12$ & 3.0 & +18.9 \\
180583 & 6190 & 2.14 & 4.8 & -0.1 & 2 & $<0.2$ & - & 7.5 & -18.9 \\
201601 & 7700 & 4.2 & 2.6 & 0.5 & 1 & $3.9 \pm 0.1$ & $50 \pm 5$ & 0.0 & -15.0 \\
\hline
\end{tabular}

${ }^{a}$ Metallicity used for the Opacity Distribution Functions, expressed as the logarithmic difference from the solar value.

${ }^{b}$ Reference for the adopted atmospheric parameters: 1 = Ryabchikova et al. (2001); 2 = Gray et al. (2001b).

${ }^{c}$ Magnetic field modulus, averaged over the visible stellar disk.

${ }^{d}$ Inclination with respect to the line of sight.

${ }^{e}$ Radial velocity.

spectrum, calculated with these oscillator strengths, plotted together with the observed spectrum.

We should point out that an iron line at $15631.386 \AA$ from the Kurucz line list with $\log (g f)=-0.399$ and $\chi_{\mathrm{e}}[\mathrm{eV}]=5.874$ is not observed and has been excluded from our models. Some weak lines present in the solar spectrum that are not reproduced by our calculations have been identified as iron lines (Biémont et al. 1985a) or as chromium lines (Biémont et al. 1985b). Unfortunately, the atomic parameters of these lines are not tabulated, so we cannot include them in our calculations. Otherwise there is generally good agreement between the observed solar spectrum and our synthetic spectrum in this wavelength region.

\subsection{Magnetic effects}

Stift \& Leone (2003) have discussed in substantial detail the effects of magnetic fields on line strengths and investigated many different kinds of Zeeman patterns. They found magnetic intensifications (i.e. increases over the zero field equivalent width) of up to a factor of 10 when the number of Zeeman components becomes very large. The shape of the surface that describes the variation of the equivalent width (EW) with magnetic field strength and field angle was shown to be a strong function of the Zeeman splitting pattern.

As stated in the introduction, we chose to study the $15625-15665 \AA$ spectral region because of the presence of the Fe I $15648.510 \AA$ line with its large effective Landé factor of $g_{\text {eff }}=2.97$. There are two more neutral iron lines within this region, located at 15652.874 and $15662.016 \AA$, whose effective Landé factors are 1.45 and 2.04, respectively. These lines can also be used for determining stellar magnetic field strengths.

We have modelled the Fe I 15648.510, 15652.874 and $15662.016 \AA$ lines for various values of the magnetic field, with the aim of investigating the behaviour of both the overall line strength (equivalent width) and the line profile as a function of the magnetic field strength. Line profiles resulting from a transverse, $45^{\circ}$ inclined and longitudinal magnetic field are shown in Fig. 3. Equivalent width variations are displayed in Fig. 4 for solar iron abundance and for a 10 times solar abundance. As seen in these figures, the Fe I $15648.510 \AA$ line appears as a simple triplet for magnetic fields of up to a few $\mathrm{kG}$. Over this range in field strength, the EW increases steadily with $B$. Beyond $B \approx 1 \mathrm{kG}$ the triplet components 

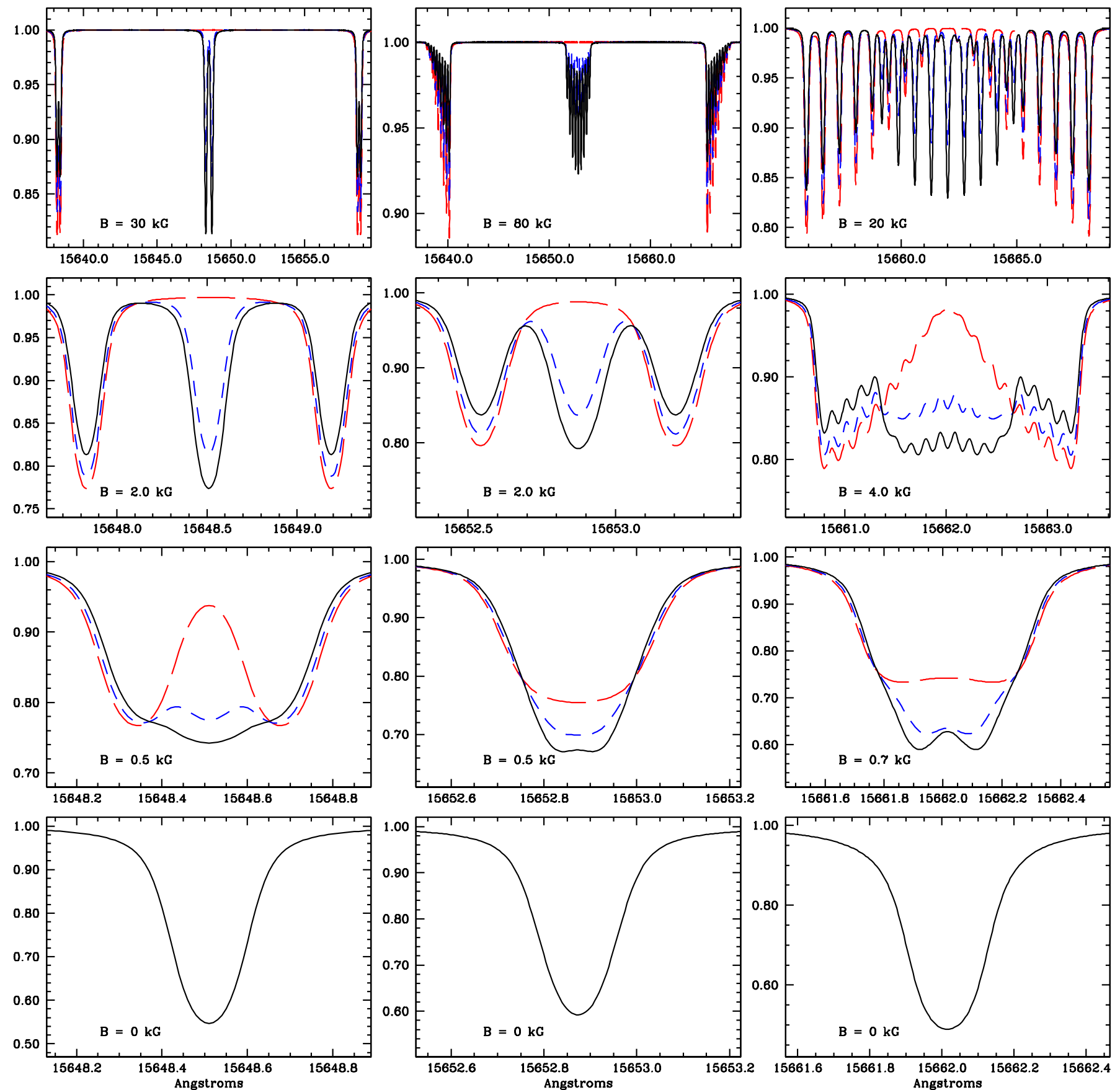

Fig. 3. Left panel: intensity (Stokes $I$ ) profile of the Fe I $15648 \AA$ line as a function of magnetic field strength. For a few KG field strengths, the line appears to be a simple triplet, but for strongest fields the $\pi$ and $\sigma$ components exhibit narrow splitting. Centre panel: the same for the Fe I $15653 \AA$ line. This line also appears to be a simple Zeeman triplet for relatively weak magnetic fields; otherwise the $\pi$ and $\sigma$ components display noticeable splitting. Right panel: the same for the Fe I $15662 \AA$ line. As opposed to the other two lines, the overlapping $\pi$ and $\sigma$ components never mimic a triplet. Long dashed lines represent a purely longitudinal magnetic field, short dashed lines a $45^{\circ}$ inclined field and solid lines a purely transverse field.

become well separated and the EW remains approximately constant. For magnetic fields of $B \geq 10 \mathrm{kG}$, the individual subcomponents of the $\pi$ and $\sigma$ components start to separate from each other and the EW increases again with the magnetic field strength. Similar behaviour is found in the Fe I $15652.874 \AA$ line. In contrast, the EW of the Fe I $15662.016 \AA$ line, whose $\pi$ and $\sigma$ components strongly overlap, increases steadily up to $B>10 \mathrm{kG}$.
Figure 5 shows the ratio of the equivalent widths of the Fe I 15648.510 and the Fe I $15652.874 \AA$ lines as a function of the magnetic field strength for both solar iron abundance and 10 times solar. Based on our numerical modelling of the magnetic intensification, it appears that this EW ratio can yield a rough estimate of the magnetic field strength, provided that an accurate and independent measure of the iron abundance is also available. However, as already noted by Gondoin et al. (1985) 


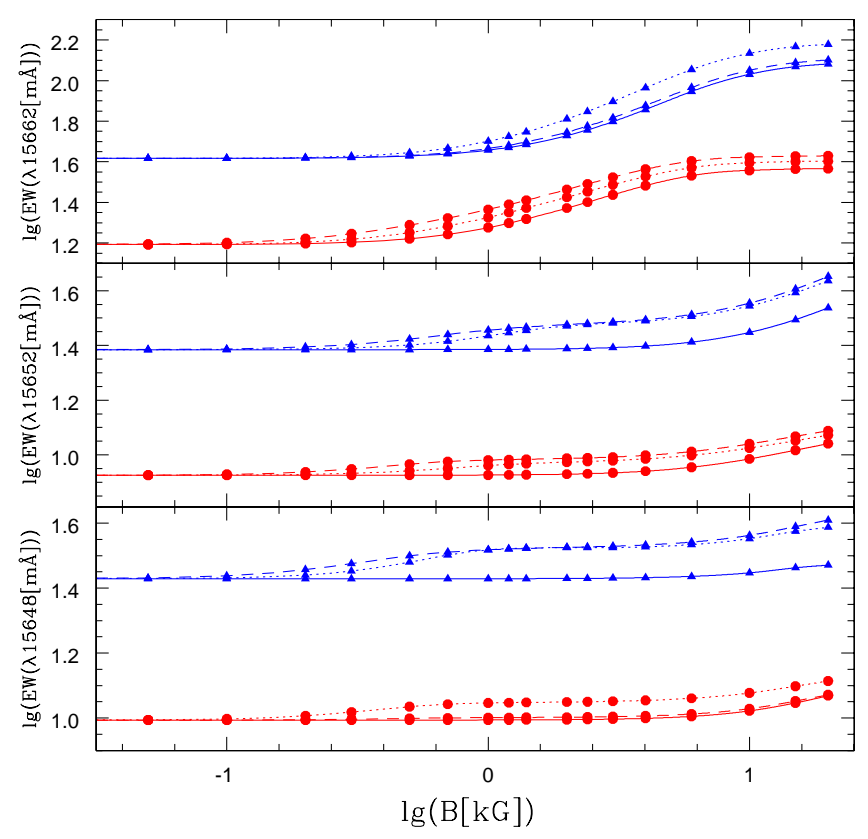

Fig. 4. The magnetic intensification (increase in equivalent width over the zero field value) of the Fe I 15648, 15653 and $15662 \AA$ lines is given for different field inclinations and two different iron abundance values, viz. $\log (\mathrm{Fe} / \mathrm{H})=-4.33$ (dots) and -3.33 (triangles). Solid lines represent a longitudinal field, dashed lines a field inclined by $30^{\circ}$ and dotted lines a purely transverse field.

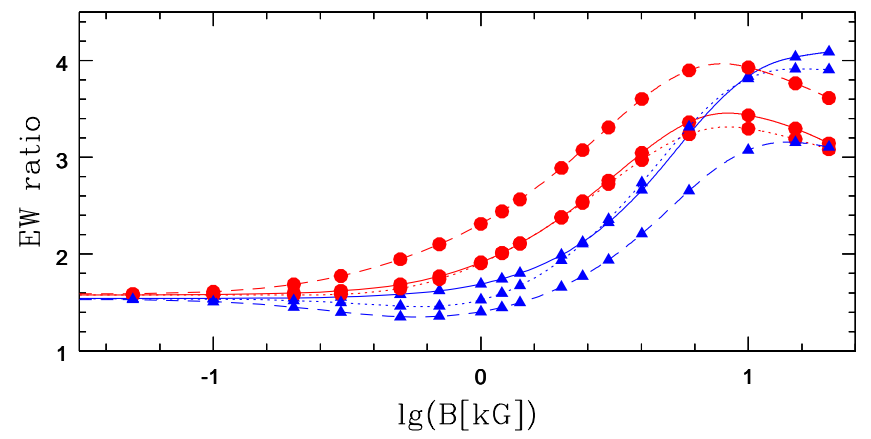

Fig. 5. Equivalent width ratios between the Fe I 15648.258 and Fe I $15652.874 \AA$ lines as a function of the magnetic field strength, for several values of field inclination and two different iron abundance values, viz. $\log (\mathrm{Fe} / \mathrm{H})=-4.33$ (dots) and $\log (\mathrm{Fe} / \mathrm{H})=-3.33$ (triangles). Solid lines represent a longitudinal field, dashed lines a field inclined by $30^{\circ}$ and dotted lines a transverse field.

and as we shall demonstrate below, line blends can severely limit the accuracy of this method.

\section{Results for individual stars}

\subsection{HD $176232=H R 7167=H I P 93179=10 \mathrm{Aql}$}

Preston (1970) estimated the rotational period of HD 176232 to be about 1 year, and he later (Preston 1971) measured a mean magnetic field modulus of $2.1 \mathrm{kG}$. Mathys \& Lanz (1992) obtained a high resolution spectrum of the Fe II $6149.2 \AA$ line and found no clear evidence of Zeeman splitting. However, they concluded that the differences in EW between the Fe II 6147.7 and Fe II $6149.2 \AA$ lines were due to the presence of a magnetic
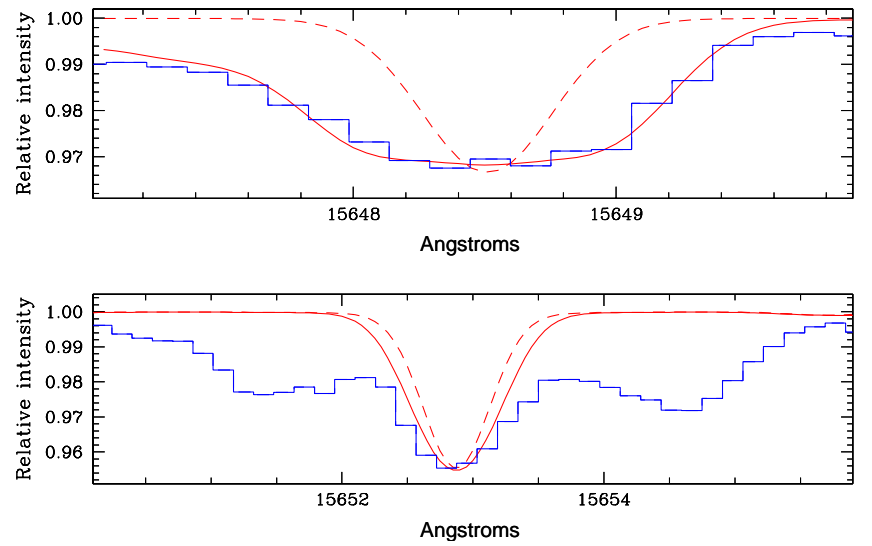

Fig. 6. Top panel: observed line profile (histogram) of Fe I 15648.235 $\AA$ in HD 176232. Dashed lines represent synthetic profiles without magnetic field. Solid lines represent synthetic profiles computed with a $1.4 \mathrm{kG}$ field inclined by $55^{\circ}$. Bottom panel: for the same magnetic field as before, the synthetic profile of the Fe I $15652.874 \AA$ line is compatible with the observed line profile when the presence of the two unidentified blending lines is taken into account.

field. By matching the Fe I $6173.34 \AA$ line profile obtained on 04 April 2001 (seven months after our near-infrared observations), Kochukhov et al. (2002) estimated the mean magnetic field modulus of HD 176232 to be about $1.5 \mathrm{kG}$ and the projected rotational velocity to be $2.0 \mathrm{~km} \mathrm{~s}^{-1}$.

For the analysis of our near-infrared spectrum of HD 176232, we adopted the atmospheric parameters given by Ryabchikova et al. (2001), listed in Table 1. Unfortunately, most of the observed near-infrared lines cannot be identified. Because of the radial velocity shift, the Fe I $15662.016 \AA$ line is not present in our spectrum (Fig. 1). From our modeling of the Fe I $15648.258 \AA$ and the Fe I $15652.874 \AA$ lines, we found that their widths cannot be matched with a zero magnetic field (Fig. 6). A magnetic field with a modulus of $\sim 1.4 \mathrm{kG}$, inclined by $\sim 55^{\circ}$ with respect to the line of sight, $\log (\mathrm{Fe} / \mathrm{H})=$ -3.98 , and a projected rotational velocity of $3.0 \mathrm{~km} \mathrm{~s}^{-1}$ are needed to match the observed profile of the Fe I $15648.258 \AA$ line. These parameters were determined by fitting the model to the observations, varying the parameters to minimize $\chi^{2}$. The uncertainties on the observed flux values at each wavelength were computed from the estimated $S / N$ values (Table 1). The errors on the fit parameters were estimated from the $\chi_{\min }^{2}+1$ surface (Press et al. 1992). A unit increment in the $\chi^{2}$ in fitting the Fe I $15648.258 \AA$ line profile gives left and right errors of $-10^{\circ}$ and $+15^{\circ}$, respectively, for the inclination angle of the field, together with a $0.1 \mathrm{kG}$ error in the field strength. We note with satisfaction that our magnetic field strength estimate is in good agreement with that of Kochukhov et al. (2002).

This field strength is also consistent with the observed profile of the Fe I $15652.874 \AA$ line (Fig. 6), although the line profile is substantially distorted by the presence of two adjacent unidentified lines. This serves to demonstrate how severe blending with unidentified lines can render some magnetically sensitive features useless for estimating magnetic field strengths. 
The radial velocity $(R V)$ of HD 176232 as measured from our spectra is $+18.9 \mathrm{~km} \mathrm{~s}^{-1}$, a value consistent with the $+18.14 \mathrm{~km} \mathrm{~s}^{-1}$ velocity measured by Carrier et al. (2002).

\section{2. $H D 180583=H R 7308=H I P 94685=V 473$ Lyr}

The star HD 180583 is classified as a possible F6 Sr by Catalano \& Renson 1998), but is in fact a cepheid (Kovtyukh et al. 2003) whose basic parameters have been determined by Gray et al. (2001a,b). From a study of cepheids based on CORAVEL data, Bersier \& Burki (1996) found that the cross-correlation function for HD 180583 gives a line broadening, due to both microturbulence and rotational velocity, of $5.3 \mathrm{~km} \mathrm{~s}^{-1}$. When combined with the microturbulence value of $4.8 \mathrm{~km} \mathrm{~s}^{-1}$ estimated by Gray et al. (2001b), this suggests that the projected rotational velocity of this star is about $5 \mathrm{~km} \mathrm{~s}^{-1}$.

Adopting the atmospheric parameters listed in Table 2, we were able to match the Fe I 15648.258, 15652.887 and $15662.016 \AA$ lines assuming a $4.8 \mathrm{~km} \mathrm{~s}^{-1}$ microturbulence, zero magnetic field strength and a $7.5 \mathrm{~km} \mathrm{~s}^{-1}$ projected rotational velocity (Fig. 7). In order to match the observed line widths an additional $2.5 \mathrm{~km} \mathrm{~s}^{-1}$ line broadening was required with respect to the projected rotational velocity estimated by Bersier \& Burki (1996). This broadening could be due to stellar oscillations even though we do not observe any systematic line asymmetry characteristic of pulsations (Fig. 7).

We tried to match the three near infrared line profiles assuming various weak magnetic field strengths, microturbulent velocities and rotational velocities. We found that the Fe I $15648.258 \AA$ A line profile computed with a $0.5 \mathrm{kG}$ field reproduces the observed profile under the assumption of zero rotational broadening; however, the profiles of the other two lines are then too narrow with respect to the observations (Fig.7). The strongest magnetic field compatible with the profiles of the three lines is $0.2 \mathrm{kG}$, with a $10 \mathrm{~km} \mathrm{~s}^{-1}$ rotational velocity. Therefore, if there is any magnetic field at all in HD 180583, the mean magnetic field modulus must be less than $0.2 \mathrm{kG}$. We conclude that HD 180583 is not a magnetic chemically peculiar star.

We measured a radial velocity of $-18.9 \mathrm{~km} \mathrm{~s}^{-1}$ for HD 180583. This value is within the range of radial velocities reported by Gorynya et al. (1998): -11.30 to $-19.40 \mathrm{~km} \mathrm{~s}^{-1}$.

\section{3. $H D 201601=H R 8097=H I P 104521=\gamma E q u$}

According to Leroy et al. (1994), the rotational axis of HD 201601 is inclined with respect to the line of sight by $150^{\circ}$. The magnetic field is represented by a centred dipole whose symmetry axis is tilted with respect to the rotational axis by $80^{\circ}$; the polar field strength is $5.5 \mathrm{kG}$. Assuming a $77 \mathrm{yr}$ period, Kochukhov \& Ryabchikova (2001) estimated the rotational velocity of HD 201601 to be $0.003 \mathrm{~km} \mathrm{~s}^{-1}$.

The magnetic field of HD 201601 is so strong and its rotational velocity so low that Zeeman splitting is visible in the Fe II $6149.258 \AA$ line (Mathys \& Lanz 1992). Our visible spectrum of HD 201601 was obtained one year after the nearinfrared spectrum; however, because of the very long rotational


Fig. 7. The Fe I $15648.258,15652.874$ and $15662.016 \AA$ line profiles (histograms) of HD 180583 can be matched with a null magnetic field assuming rotational velocity of $7.5 \mathrm{~km} \mathrm{~s}^{-1}$. Dashed lines represent line profiles computed for a $0.5 \mathrm{kG}$ field, null microturbulence and no rotational broadening.

period of this star, the two spectra correspond to fairly similar rotational phases.

Because of the rather strong magnetic field in HD 201601, the Zeeman components of the near-infrared lines are expected to exhibit fairly large separations. However, due to the relatively high effective temperature of $7700 \mathrm{~K}$, the near-infrared lines of this star should be very weak, similar in strength to those seen in the spectrum of HD 176232. Indeed, we find that the lines in the near-infrared spectrum are no deeper than $4 \%$ of the continuum (Fig. 8). In addition, a large number of spectral lines remains unidentified, which, unfortunately, makes it extremely difficult to infer the magnetic field strength and geometry by modeling only the observed near-infrared spectrum.

We checked whether the magnetic field inferred from the optical spectrum is consistent with the observed near-infrared spectrum, adopting for this purpose the stellar parameters given in Table 1. From the separation of the Zeeman components in the Fe II $6149.258 \AA$ Alne we obtained an estimate for the mean magnetic field modulus of $3.9 \pm 0.1 \mathrm{kG}$, in excellent agreement with the value of $3846 \pm 59 \mathrm{G}$ found by Mathys et al. (1997). Rotational broadening being virtually absent, we had to invoke some extra broadening mechanism in order to explain the observed widths of the metal lines in HD 201601. Non-radial oscillations or element stratification are possibilities (Ryabchikova et al. 2002) but for our present purpose we simply assumed a $2.6 \mathrm{~km} \mathrm{~s}^{-1}$ microturbulence that reproduces the shape of the Zeeman components in the Fe II 6149.258 line fairly well. The iron abundance derived with this microturbulence value is $\log (\mathrm{Fe} / \mathrm{H})=-4.43$.

Modeling the ratio of the relative strengths of the $\pi$ and the $\sigma$ components - which depends on the angle between the 

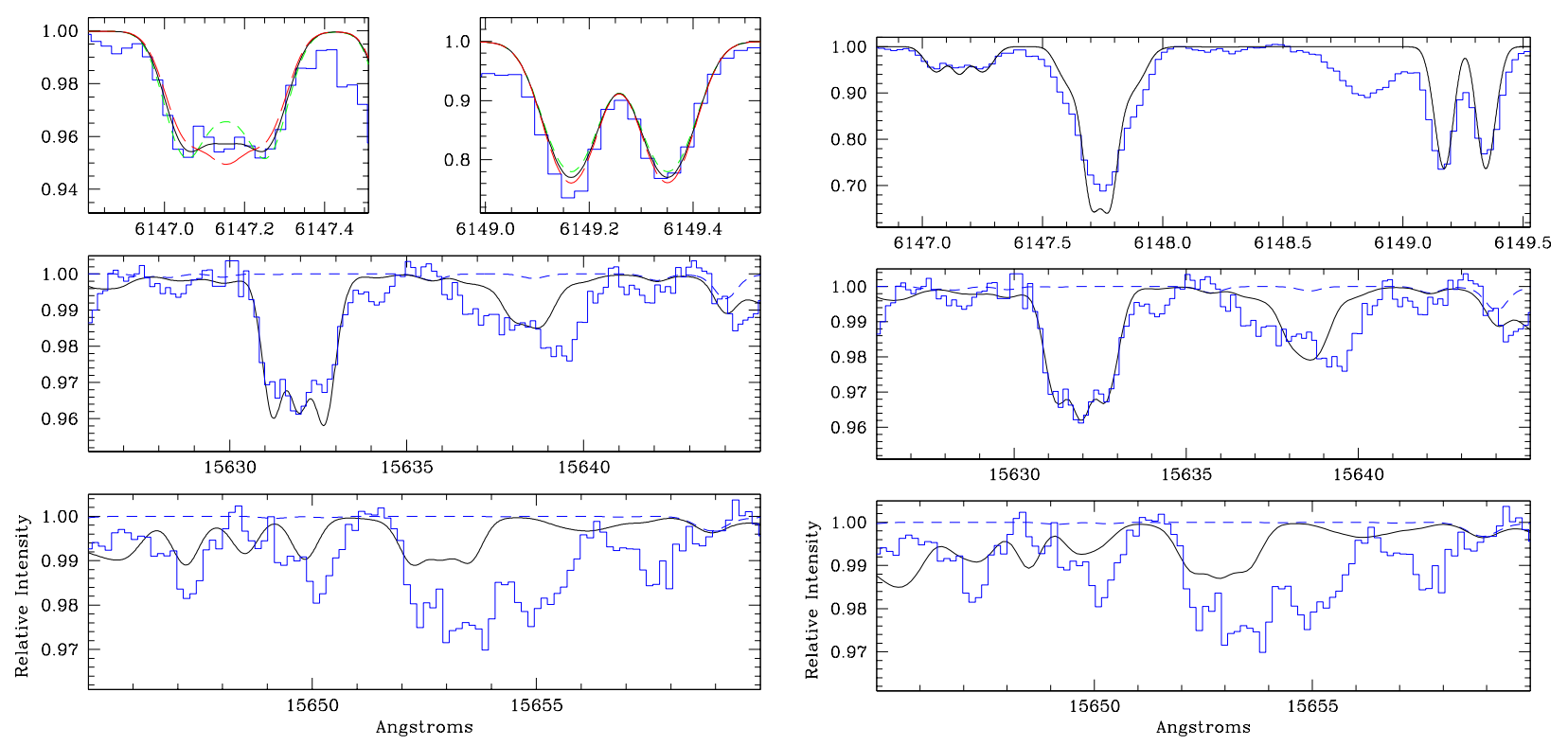

Fig. 8. Left panel: portion of the spectrum of HD 201601 in the visible (histogram) containing the Cr II $6147.1 \AA$ line; the relative intensities of the $\pi$ and the $\sigma$ components have been used to estimate the inclination of the magnetic field. We display the synthetic COSSAM spectrum assuming an inclination of $50^{\circ}$ (full line), $40^{\circ}$ (short dashed line), and $60^{\circ}$ (long dashed). The highly Zeeman sensitive Fe II $6149.3 \AA$ line has been used for estimating a $3.9 \mathrm{kG}$ mean field modulus. The observed near-IR spectrum (histogram) is plotted together with the synthetic spectrum for $50^{\circ}$ inclination (solid line). The dashed line represents the predicted telluric spectrum. Right panel: comparison of the observed spectra with the dipole configuration (solid line) given by Leroy et al. (1994).

direction of the magnetic field and the line of sight - in the Cr II 6147.1 $\AA$ line, we found that the average field inclination is $50^{\circ} \pm 5^{\circ}$ (top left panel in Fig. 8). The chromium abundance is determined to be $\log (\mathrm{Cr} / \mathrm{H})=-5.28$. It should be pointed out that the Fe II $6149.258 \AA$ line cannot be used to determine the magnetic field direction since the $\pi$ and the $\sigma$ components coincide in wavelength.

We estimated a radial velocity for HD 201601 of $-15.0 \mathrm{~km} \mathrm{~s}^{-1}$, a value within the range of velocities quoted in the literature: $R V=-12.25 \mathrm{~km} \mathrm{~s}^{-1}(\mathrm{JD}=2450297.5454)$ and $R V=-4.28(\mathrm{JD}=2450356.4434)$ measured by Hildebrandt et al. (2000), $R V=-16.37 \mathrm{~km} \mathrm{~s}^{-1}(\mathrm{JD}=2451404.530)$ measured by Ersparmer \& North (2003).

With the magnetic field strength and direction derived from the spectral lines in the optical spectrum, we computed the synthetic near-infrared spectrum of HD 201601. For an abundance of $\log (\mathrm{Fe} / \mathrm{H})=-4.43$, the synthetic iron lines are too strong with respect to the observations, so we adopted an iron abundance value of -4.53 .

Figure 8 shows that differences between the synthetic and observed spectra are within the errors, even though there are a large number of unidentified spectral lines in the observed spectrum. We also computed a synthetic spectrum assuming the dipole geometry suggested by Leroy (1994) (right panel of Fig. 8). Leroy et al. (1994) determined the dipolar configuration of HD 210601 on the basis of broadband linear polarisation and variability of the mean longitudinal magnetic field; therefore, higher terms in the multipolar expansion (i.e. beyond the dipole) which describe the small scale spatial variation of the field, could not be determined. These higher terms, representing quadrupolar or even higher multipolar components of the magnetic field, could well be important in shaping the line profiles and hence may be responsible for the differences between the observed optical and near-infrared spectra and our synthetic spectrum (computed with the assumption of a dipole field). Unidentified spectral lines are also certainly present in both the visible and near-infrared spectra.

\section{Conclusions}

We have obtained high resolution spectra in the $15625-15665 \AA$ near-infrared region of the two MCP star HD 176232 and HD 201601 as well as the suspected MCP star HD 180583. For the three iron lines that dominate this region we have computed the line profiles and the increases in EW (the magnetic intensification) for magnetic field strengths up to $80 \mathrm{kG}$. We confirm that EW ratios of these lines could in principle provide a means for deducing the magnetic field strength (for a detailed discussion of magnetic intensification as a function of Zeeman pattern, magnetic field strength and field angle, we refer to Stift \& Leone 2003). However, as it turns out, the EW ratios depend not only on abundance and on field geometry, but are also very sensitive to line blending (Gondoin et al. 1985), which leads us to the conclusion that spectral synthesis is the only reliable tool for the study of stellar magnetic fields.

From the comparison between computed and observed line profiles, we find that the mean magnetic field modulus of the chemically peculiar star HD 176232 is about $1.4 \mathrm{kG}$. For the suspected magnetic chemically peculiar star (and unusual cepheid) HD 180583, it is possible to match the observed profiles without a magnetic field; a possible field must be much weaker than $0.2 \mathrm{kG}$.

The observed near-infrared spectrum of HD 201601, a star characterised by a rather strong magnetic field and a relatively 
high effective temperature, exhibits features so weak that they cannot be used for magnetic field determinations. Furthermore, most of the observed spectral features have not been conclusively identified. Nevertheless, we find that, within the errors, the magnetic field strength and direction inferred from spectral lines in the optical are consistent with our observed nearinfrared spectrum. We note that higher multipole terms in the magnetic field could be important in shaping the observed line profiles.

We conclude that high-resolution, high, signal-to-noise spectroscopy of near-infrared lines can be very useful in determining stellar magnetic field geometry and strength, particularly in cool stars. The analysis of the profiles of these lines is probably the best diagnostic method for inferring the presence of weak magnetic fields. When magnetic fields are large, the Zeeman splitting in the near infrared can grow so large that the various Zeeman components of different lines become blended. The analysis of near-infrared lines in stars of relatively high temperature (such as the MCP star HD 201601) however runs into serious difficulties, as the depth of these lines are of the order of $4 \%$ at most.

The detection and measurement of stellar magnetic fields using near-infrared lines has been limited in the past by the low resolution of the spectrographs, which in turn was mainly due to the low sensitivity of the detectors. Now that we can carry out high resolution spectroscopy in the near infrared thanks to a new generation of high-sensitivity detectors, and that with high performance multi-processor computers the modelling of magnetically split spectral lines in magnetic chemically peculiar stars has become a computation requiring only a matter of minutes to hours rather than of hours to days, we are at present limited by the lack of extensive and reliable atomic line data in the near infrared. We strongly advocate an effort towards the identification of lines in the near infrared and the determination of reliable atomic data for these lines. Only then shall we be able to take full advantage of modern near-infrared technology in the observation of stellar magnetic fields, which play such an important role in so many objects across the HR diagram.

Acknowledgements. This research is based upon work supported by the National Aeronautics and Space Administration under Cooperative Agreement no. NCC 5-538 issued through the Office of Space Science, Planetary Astronomy Program. This work has also made use of observations obtained with the Italian Telescopio Nazionale Galileo (TNG) operated on the island of La Palma by the Centro Galileo Galilei of the INAF (Istituto Nazionale di Astrofisica) at the Spanish Observatorio del Roque de los Muchachos of the Instituto de Astrofisica de Canarias. NSO/Kitt Peak FTS data used here were produced by NSF/NOAO. MJS gratefully acknowledges support by the Austrian Science Fund (FWF), project P16003-N05. WDV would like to thank James Graham for support.

\section{References}

Auer, L. H., Heasley, J. N., \& House, L. L. 1977, ApJ, 216, 531

Babcock, H. W. 1949, Observatory, 69, 191

Babcock, H. W. 1958, ApJS, 3, 141

Biémont, E., Roland, G., Delbouille, L., \& Brault, J. W. 1985a, A\&AS, 61, 107
Biémont, E., Delbouille, L., Roland, G., \& Brault, J. W. 1985b, A\&AS, 61, 185

Bersier, D., \& Burki, G. 1996, A\&A, 306, 417

Carrier, F., North, P., Udry, S., \& Babel, J. 2002, A\&A, 394, 151

Castelli, F., \& Kurucz, R. L. 1994, A\&A, 281, 817

Catalano, F. A., \& Renson, P. 1998, A\&AS, 127, 421

Erspamer, D., \& North, P. 2003, A\&A, 398, 1121

Gondoin, Ph., Giampapa, M. S., \& Bookbinder, J. A. 1985, ApJ, 297, 710

Goodson, A. P., Böhm, K.-H., \& Winglee, R. M. 1999, ApJ, 524, 142

Gorynya, N. A., Samus', N. N., Sachkov, M. E., et al. 1998, PAZh, 24, 939

Gratton, R., Bonanno, G., Bruno, P., et al. 2003, ApSS, in press

Gray, R. O., Napier, M. G., \& Winkler, L. I. 2001a, AJ, 121, 2148

Gray, R. O., Graham, P. W., \& Hoyt, S. R. 2001b, AJ, 121, 2159

Greene, T. P., Tokunaga, A. T., Toomey, D. W., \& Carr, J. B. 1993, SPIE, 1946, 313

Harvey, J., \& Hall, D. 1975, BAAS, 7, 459

Hildebrandt, G., Scholz, G., \& Lehmann, H. 2000, AN, 321, 115

Kochukhov, O., Landstreet, J. D., Ryabchikova, T., Weiss, W. W., \& Kupka, F. 2002, MNRAS, 337, L1

Kochukhov, O., \& Ryabchikova, T. 2001, A\&A, 377, 22

Kovtyukh, V. V., Andrievsky, S. M., Luck, R.E., \& Gorlova, N. I. 2003, A\&A, 401, 661

Kurucz, R. L. 1993, A new opacity-sampling model atmosphere program for arbitrary abundances, in: IAU Coll. 138, Peculiar versus normal phenomena in A-type and related stars, ed. M. M. Dworetsky, F. Castelli, \& R. Faraggiana, ASP Conf. Ser., 44, 87

Kurucz, R. L. 1995, Atomic Line Data (R. L. Kurucz and B. Bell) Kurucz CD-ROM No. 23 (Cambridge, Mass.: Smithsonian Astrophysical Observatory)

Landstreet, J. D., \& Mathys, G. 2000, A\&A, 359, 213

Leone, F., \& Manfrè, M. 1996, A\&A, 315, 526

Leroy, J. L., Bagnulo, S., Landolfi, M., \& Degli'Innocenti, E. L. 1994, A\&A, 284, 174

Liebert, J. Bergeron, P., \& Holberg, J. B. 2003, AJ., 125, 348

Lord, S. D. 1992, NASA Technical Memor. 103957

Mathys, G., \& Lanz, T. 1992, A\&A, 256, 169

Mathys, G., Hubrig, S., Landstreet, J. D., Lanz, T., \& Manfroid, J. 1997, A\&AS, 123, 353

Melendez, J., \& Barbuy, B. 1999, ApJS, 124, 527

Michaud, G. 1970, ApJ, 160, 641

Montesinos, B., Thomas, J. H., Ventura, P., \& Mazzitelli, I. 2001, MNRAS, 326, 877

Press, W. H., Teukolsky, S. A., Vetterling, W. T., \& Flannery, B. F. 1992, Numerical Recipes, 2nd ed. (Cambridge University Press)

Preston, G. W. 1970, PASP, 82, 878

Preston, G. W. 1971, ApJ, 164, 309

Rauw, G., Blomme, R., Waldron, W. L., et al. 2002, A\&A, 394, 993

Renson, P., Gerbaldi, M., \& Catalano, F. A. 1991, A\&AS, 89, 429

Ryabchikova, T. A., Savanov, I. S., Malanushenko, V. P., \& Kudryavtsev, D. O. 2001, Astron. Rep., 45, 382

Ryabchikova, T., Piskunov, N., Kochukhov, O., et al. 2002, A\&A, 384, 545

Robinson, R. D. 1980, ApJ, 239, 961

Saar, S. H. 1994, Infrared Measurements of Stellar Magnetic Fields, in Infrared Solar Physics, IAU Symp. 154, ed. D. M. Rabin, J. T. Jefferies, \& C. Lindsey (Dordrecht: Kluwer Academic Publishers)

Solanki, S. K. 1994, Properties of Magnetic Features from the Analysis of Near-Infrared Spectral Lines, in Infrared Solar Physics, IAU Symp. 154, ed. D. M. Rabin, J. T. Jefferies, \& C. Lindsey (Dordrecht: Kluwer Academic Publishers)

Stift, M. J. 2000, A Peculiar Newsletter, 33

Stift, M. J., \& Leone, F. 2003, A\&A, 398, 411

Wade, G. A., Bagnulo, S., Kochukhov, O., et al. 2001, A\&A, 374, 265 